United Nations Educational Scientific and Cultural Organization and International Atomic Energy Agency

THE ABDUS SALAM INTERNATIONAL CENTRE FOR THEORETICAL PHYSICS

\title{
ROUGH CONVERGENCE IN NORMED LINEAR SPACES
}

\author{
H. X. $\mathrm{Phu}^{1}$ \\ Institute of Mathematics, P.O.Box 631 Bo Ho, 10000 Hanoi, Vietnam \\ and \\ The Abdus Salam International Centre for Theoretical Physics, Trieste, Italy.
}

\begin{abstract}
$x_{*} \in X$ is said to be an $r$-limit point of a sequence $\left(x_{i}\right)$ in some normed linear space $(X,\|\cdot\|)$ if $\lim \sup \left\|x_{i}-x_{*}\right\| \leq r(r \geq 0)$. The set of all $r$-limit points of $\left(x_{i}\right)$, denoted by $\operatorname{LIM}^{r} x_{i}$, is bounded, closed and convex. This paper deals with further properties of $\operatorname{LIM}^{r} x_{i}$ in case $X=\mathbb{R}^{n}$, in particular, with the relation between this rough convergence and other convergence notions, and with the dependence of $\operatorname{LIM}^{r} x_{i}$ on the roughness degree $r$. For instance, the set-valued mapping $r \mapsto \operatorname{LIM}^{r} x_{i}$ is strictly increasing and continuous on $(\bar{r},+\infty)$, where $\bar{r}:=\inf \left\{r \in \mathbb{R}_{+}: \operatorname{LIM}^{r} x_{i} \neq \emptyset\right\}$. For a so-called $\rho$-Cauchy sequence $\left(x_{i}\right)$ satisfying

$$
\forall \varepsilon>0 \quad \exists i_{\varepsilon}: i, j \geq i_{\varepsilon} \Rightarrow\left\|x_{i}-x_{j}\right\|<\rho+\varepsilon,
$$
\end{abstract}

it is shown that in general $r=\frac{n}{n+1} \rho$ (or $r=\sqrt{\frac{n}{2(n+1)}} \rho$ for Euclidean space) is the best convergence degree such that $\operatorname{LIM}^{r} x_{i} \neq \emptyset$.

$$
\text { MIRAMARE - TRIESTE }
$$

July 2000

\footnotetext{
1 Regular Associate of the Abdus Salam ICTP.
} 


\section{INTRODUCTION}

Let $\left(x_{i}\right)$ be a sequence in some normed linear space $(X,\|\cdot\|)$, and $r$ be a nonnegative real number. $\left(x_{i}\right)$ is said to be $r$-convergent to $x_{*}$, denoted by $x_{i} \stackrel{r}{\rightarrow} x_{*}$, if

$$
\forall \varepsilon>0 \quad \exists i_{\varepsilon} \in \mathbb{N}: i \geq i_{\varepsilon} \Rightarrow\left\|x_{i}-x_{*}\right\|<r+\varepsilon,
$$

or equivalently, if

$$
\lim \sup \left\|x_{i}-x_{*}\right\| \leq r
$$

This is the rough convergence with $r$ as roughness degree. For $r=0$ we have the classical convergence again. But our proper interest is case $r>0$. There are several reasons for this interest. For instance, since an originally convergent sequence $\left(y_{i}\right)$ (with $y_{i} \rightarrow x_{*}$ ) often cannot be determined (i.e., measured or calculated) exactly, one has to do with an approximated sequence $\left(x_{i}\right)$ satisfying $\left\|x_{i}-y_{i}\right\| \leq r$ for all $i$, where $r>0$ is the maximal approximation error. Then $\left(x_{i}\right)$ is no more convergent in the classical sense, but

$$
\left\|x_{i}-x_{*}\right\| \leq\left\|x_{i}-y_{i}\right\|+\left\|y_{i}-x_{*}\right\| \leq r+\left\|y_{i}-x_{*}\right\|
$$

implies that it is $r$-convergent in the sense of (1.1).

If (1.1) holds, $x_{*}$ is an $r$-limit point of $\left(x_{i}\right)$, which is usually no more unique (for $r>0$ ). So we have to consider the so-called $r$-limit set (or shortly: $r$-limit) of $\left(x_{i}\right)$ defined by

$$
\operatorname{LIM}^{r} x_{i}:=\left\{x_{*} \in X: x_{i} \stackrel{r}{\rightarrow} x_{*}\right\} .
$$

$\left(x_{i}\right)$ is said to be $r$-convergent if $\operatorname{LIM}^{r} x_{i} \neq \emptyset$. In this case, $r$ is also called a convergence degree of $\left(x_{i}\right)$.

Let us illustrate by an example. Clearly, the sequence $\left(y_{i}\right)$ with

$$
y_{i}=0.5+2(-1)^{i} / i, \quad i=1,2, \ldots
$$

converges to 0.5 . For sufficiently large $i$, it is impossible to calculate $y_{i}$ exactly by computer, but it is rounded to some machine number, e.g. to the nearest one. For the sake of simplicity, we normalize so that $y_{i}$ is approximated by

$$
x_{i}:=\operatorname{rd}\left(y_{i}\right):=z \quad \text { where } z \text { is the integer satisfying } z-0.5 \leq y_{i}<z+0.5
$$

Then

$$
x_{1}=-1, \quad x_{2}=2, \quad x_{2 j-1}=0 \text { and } x_{2 j}=1 \text { for } j=2,3, \ldots
$$

Obviously, the sequence $\left(x_{i}\right)$ does not converge anymore. But by definition we have $x_{i} \stackrel{r}{\rightarrow} 0.5$ for $r=0.5$, and altogether

$$
\operatorname{LIM}^{r} x_{i}= \begin{cases}\emptyset & \text { for } r<0.5 \\ {[1-r, r]} & \text { for } r \geq 0.5\end{cases}
$$


Sometimes we are interested in the set of $r$-limit points lying in a given subset $S \subset X$, which is called $r$-limit in $S$ and denoted by

$$
\operatorname{LIM}^{S, r} x_{i}:=\left\{x_{*} \in S: x_{i} \stackrel{r}{\rightarrow} x_{*}\right\}
$$

(Obviously, $\operatorname{LIM}^{X, r} x_{i}=\operatorname{LIM}^{r} x_{i}$ and $\operatorname{LIM}^{S, r} x_{i}=S \cap \operatorname{LIM}^{r} x_{i}$.) For instance, with $S=\left(x_{i}\right)$, the set of the $r$-limit points of $\left(x_{i}\right)$ lying in this sequence is considered, which is rather unusual in the classical point of view. In the example given by (1.4)-(1.5), we have

$$
\operatorname{LIM}^{\left(x_{i}\right), r} x_{i}= \begin{cases}\emptyset & \text { for } r<1 \\ \left\{x_{i}: i \geq 3\right\} & \text { for } r \geq 1\end{cases}
$$

Similarly as above, if some Cauchy sequence $\left(y_{i}\right)$ cannot be determined exactly but is only approximated by a sequence $\left(x_{i}\right)$ with $\rho / 2>0$ as maximal approximation error, i.e., $\left\|x_{i}-y_{i}\right\| \leq \rho / 2$ for all $i$, then $\left(x_{i}\right)$ does not satisfy the classical Cauchy condition, but only fulfills the following rough Cauchy condition

$$
\forall \varepsilon>0 \exists i_{\varepsilon}: i, j \geq i_{\varepsilon} \Rightarrow\left\|x_{i}-x_{j}\right\|<\rho+\varepsilon
$$

Such a sequence is said to be a rough Cauchy sequence with roughness degree $\rho$, or $\rho$-Cauchy sequence for short. $\rho$ is also called a Cauchy degree of $\left(x_{i}\right)$.

Section 2 is devoted to some basic properties such as boundedness, closedness, convexity... of $r$-limit sets. In Section 3 the relation between rough convergence and classical convergence or set convergence is investigated. The dependence of $r$ limit on the roughness degree $r$ is the subject of Section 3. Section 4 is concerned with rough Cauchy sequences, in particular, with the relation between Cauchy degree and convergence degree of a sequence.

For the sake of simplicity, assume throughout this paper that $X=\mathbb{R}^{n}$.

\section{Elementary Properties}

First, let us transform some properties of classical convergence to rough convergence. It is well known if a sequence converges then its limit is unique. This property is not maintained for rough convergence with roughness degree $r>0$, but only has the following analogy.

Proposition 2.1. The diameter of a r-limit set is not greater than $2 r$. In general, there is no smaller bound.

Proof. We have to show that

$$
\operatorname{diam}\left(\operatorname{LIM}^{r} x_{i}\right)=\sup \left\{\|y-z\|: y, z \in \operatorname{LIM}^{r} x_{i}\right\} \leq 2 r
$$


Assume the contrary that $\operatorname{diam}\left(\operatorname{LIM}^{r} x_{i}\right)>2 r$, then there exist $y, z \in \operatorname{LIM}^{r} x_{i}$ satisfying $d:=\|y-z\|>2 r$. For an arbitrary $\varepsilon \in(0, d / 2-r)$, it follows from (1.1) and (1.3) that there is an $i_{\varepsilon} \in \mathbb{N}$ such that

$$
\left\|x_{i}-y\right\|<r+\varepsilon \text { and }\left\|x_{i}-z\right\|<r+\varepsilon \text { for } i \geq i_{\varepsilon} .
$$

This implies

$$
\|y-z\| \leq\left\|x_{i}-y\right\|+\left\|x_{i}-z\right\|<2(r+\varepsilon)<2 r+2(d / 2-r)=d,
$$

which conflicts with $d=\|y-z\|$. Hence, (2.1) must be true.

Consider a convergent sequence $\left(x_{i}\right)$ with $\lim x_{i}=x_{*}$. Then, for

$$
\bar{B}_{r}\left(x_{*}\right):=\left\{y \in X:\left\|y-x_{*}\right\| \leq r\right\}
$$

it follows from

$$
\left\|x_{i}-y\right\| \leq\left\|x_{i}-x_{*}\right\|+\left\|x_{*}-y\right\| \leq\left\|x_{i}-x_{*}\right\|+r \quad \text { for } \quad y \in \bar{B}_{r}\left(x_{*}\right)
$$

(1.1), and (1.3) that $\operatorname{LIM}^{r} x_{i}=\bar{B}_{r}\left(x_{*}\right)$. Since diam $\bar{B}_{r}\left(x_{*}\right)=2 r$, this shows that in general the upper bound $2 r$ of the diameter of an $r$-limit set cannot be decreased anymore.

Obviously, the uniqueness of limit (of classical convergence) can be regarded as a special case of the latter property, because if $r=0$ then $\operatorname{diam}\left(\operatorname{LIM}^{r} x_{i}\right)=2 r=0$, i.e., $\operatorname{LIM}^{r} x_{i}$ is either empty or a singleton.

A further property of classical concept is the boundedness of convergent sequences. Its analogy for rough convergence is:

Proposition 2.2. A sequence $\left(x_{i}\right)$ is bounded if and only if there exists an $r \geq 0$ such that $\operatorname{LIM}^{r} x_{i} \neq \emptyset$. For all $r>0$, a bounded sequence $\left(x_{i}\right)$ always contains a subsequence $\left(x_{i_{j}}\right)$ with

$$
\operatorname{LIM}^{\left(x_{i_{j}}\right), r} x_{i_{j}} \neq \emptyset
$$

Proof. If $s:=\sup \left\{\left\|x_{i}\right\|: i \in \mathbb{N}\right\}<\infty$ then $\operatorname{LIM}^{s} x_{i}$ contains the origin of $X$. On the other hand, if $\operatorname{LIM}^{r} x_{i} \neq \emptyset$ for some $r \geq 0$ then all but finite elements $x_{i}$ are contained in some ball with any radius greater than $r$. Therefore, the sequence $\left(x_{i}\right)$ is bounded.

As $\left(x_{i}\right)$ is a bounded sequence in a finite-dimensional normed space, it certainly contains a convergent subsequence $\left(x_{i_{j}}\right)$. Let $x_{*}$ be its limit point, then $\operatorname{LIM}^{r} x_{i_{j}}=\bar{B}_{r}\left(x_{*}\right)$ and, for $r>0$,

$$
\operatorname{LIM}^{\left(x_{i_{j}}\right), r} x_{i_{j}}=\left\{x_{i_{j}}:\left\|x_{*}-x_{i_{j}}\right\| \leq r\right\} \neq \emptyset .
$$


Note that the second part of the previous proposition is concerned with $r$-limit points lying in the subsequence $\left(x_{i_{j}}\right)$. It is straightforward that a sequence contained in some bounded set $S$ always possesses a subsequence being $r$-convergent (for an arbitrary $r>0$ ) to some point of $S$. Here, the closedness of $S$ is not needed as for classical convergence.

Corresponding to the property that each subsequence of a convergent sequence also converges to the same limit point, we now have the following one whose proof is rather simple.

Proposition 2.3. If $\left(x_{i}^{\prime}\right)$ is a subsequence of $\left(x_{i}\right)$, then $\operatorname{LIM}^{r} x_{i} \subseteq \operatorname{LIM}^{r} x_{i}^{\prime}$.

Those are possibly all properties having an analogy in classical concept. It is really unimaginable to say something more about properties of classical limit sets which are either empty or contain only a single point. But for rough convergence, the $r$-limit set is generally no more a singleton. So its topological and geometrical properties are of interest, as given in the next propositions.

Proposition 2.4. For all $r \geq 0$, the $r$-limit set $\mathrm{LIM}^{r} x_{i}$ of an arbitrary sequence $\left(x_{i}\right)$ is closed.

Proof. Let $\left(y_{j}\right)$ be an arbitrary sequence in $\operatorname{LIM}^{r} x_{i}$ which converges to some point $y_{*}$. For each $\varepsilon>0$, by definition, there are a $j_{\varepsilon / 2}$ and an $i_{\varepsilon / 2}$ such that $\left\|y_{j_{\varepsilon / 2}}-y_{*}\right\|<\varepsilon / 2$ and $\left\|x_{i}-y_{j_{\varepsilon / 2}}\right\|<r+\varepsilon / 2$ whenever $i \geq i_{\varepsilon / 2}$. Consequently,

$$
\left\|x_{i}-y_{*}\right\| \leq\left\|x_{i}-y_{j_{\varepsilon / 2}}\right\|+\left\|y_{j_{\varepsilon / 2}}-y_{*}\right\|<r+\varepsilon \text { if } i \geq i_{\varepsilon / 2} .
$$

That means $y_{*} \in \operatorname{LIM}^{r} x_{i}$, too. Hence, $\operatorname{LIM}^{r} x_{i}$ is closed.

Proposition 2.5

(a) If $y_{0} \in \operatorname{LIM}^{r_{0}} x_{i}$ and $y_{1} \in \operatorname{LIM}^{r_{1}} x_{i}$ then

$$
y_{\lambda}:=(1-\lambda) y_{0}+\lambda y_{1} \in \operatorname{LIM}^{(1-\lambda) r_{0}+\lambda r_{1}} x_{i}, \text { for } \lambda \in[0,1] \text {. }
$$

(b) $\operatorname{LIM}^{r} x_{i}$ is convex. If $(X,\|\cdot\|)$ is a finite-dimensional strictly convex space (i.e., the closed unit ball is strictly convex) then $\mathrm{LIM}^{r} x_{i}$ is strictly convex, i.e., $y_{0}, y_{1} \in \operatorname{LIM}^{r} x_{i}$ and $y_{0} \neq y_{1}$ imply

$$
y_{\lambda} \in \operatorname{int}\left(\operatorname{LIM}^{r} x_{i}\right) \quad \text { for all } \lambda \in(0,1) \text {. }
$$

Proof. (a) By definition, for every $\varepsilon>0$ there exists an $i_{\varepsilon}$ such that $i \geq i_{\varepsilon}$ implies $\left\|x_{i}-y_{0}\right\|<r_{0}+\varepsilon$ and $\left\|x_{i}-y_{1}\right\|<r_{1}+\varepsilon$, which yields also

$$
\begin{aligned}
\left\|x_{i}-y_{\lambda}\right\| & \leq(1-\lambda)\left\|x_{i}-y_{0}\right\|+\lambda\left\|x_{i}-y_{1}\right\| \\
& <(1-\lambda)\left(r_{0}+\varepsilon\right)+\lambda\left(r_{1}+\varepsilon\right) \\
& =(1-\lambda) r_{0}+\lambda r_{1}+\varepsilon .
\end{aligned}
$$


Hence, $y_{\lambda} \in \operatorname{LIM}^{(1-\lambda) r_{0}+\lambda r_{1}} x_{i}$.

(b) In particular, for $r=r_{0}=r_{1}$, (a) yields immediately that $\operatorname{LIM}^{r} x_{i}$ is convex.

Assume the space considered is strictly convex. In order to prove the strict convexity of $\operatorname{LIM}^{r} x_{i}$ it suffices to show that $y_{0}, y_{1} \in \operatorname{LIM}^{r} x_{i}$ and $y_{0} \neq y_{1}$ imply

$$
y_{0.5}=\frac{1}{2}\left(y_{0}+y_{1}\right) \in \operatorname{int}\left(\operatorname{LIM}^{r} x_{i}\right)
$$

because for each $y_{\lambda}, 0<\lambda<1$, there exist $y_{0}^{\prime}, y_{1}^{\prime} \in \operatorname{LIM}^{r} x_{i}$ satisfying $y_{0}^{\prime} \neq y_{1}^{\prime}$ and $y_{\lambda}=\frac{1}{2}\left(y_{0}^{\prime}+y_{1}^{\prime}\right)$.

Let $C$ be the set of cluster points of $\left(x_{i}\right)$ which is obviously closed. Moreover, since the normed space considered is finite-dimensional and $\left(x_{i}\right)$ is bounded (by Proposition 2.2), $C$ is nonempty and bounded. Therefore, there exists a $\bar{c} \in C$ satisfying

$$
\left\|\bar{c}-y_{0.5}\right\|=\max _{c \in C}\left\|c-y_{0.5}\right\| .
$$

It follows from $y_{0}, y_{1} \in \operatorname{LIM}^{r} x_{i}$ that

$$
\left\|\bar{c}-y_{0}\right\| \leq r \quad \text { and } \quad\left\|\bar{c}-y_{1}\right\| \leq r
$$

(compare (3.2)). These inequalities imply by the strict convexity of the space considered

$$
\left\|\bar{c}-y_{0.5}\right\|=\left\|0.5\left(\bar{c}-y_{0}\right)+0.5\left(\bar{c}-y_{1}\right)\right\|<\max \left\{\left\|\bar{c}-y_{0}\right\|,\left\|\bar{c}-y_{1}\right\|\right\} \leq r
$$

and thus $\sigma:=r-\left\|\bar{c}-y_{0.5}\right\|>0$. Now we have for all $c \in C$ and $y \in B_{\sigma}\left(y_{0.5}\right)$

$$
\|c-y\| \leq\left\|c-y_{0.5}\right\|+\left\|y_{0.5}-y\right\| \leq\left\|\bar{c}-y_{0.5}\right\|+\sigma=r
$$

which implies by definition $y \in \operatorname{LIM}^{r} x_{i}$. That means $y_{0.5}$ is an interior point of $\operatorname{LIM}^{r} x_{i}$.

\section{Relation to Other Convergence Notions}

In this section we investigate the relation between rough convergence and some other convergence notions, such as classical convergence and set convergence.

First, let us formulate an additive property of rough convergence.

Proposition 3.1. Suppose $r_{1} \geq 0$ and $r_{2}>0$. A sequence $\left(x_{i}\right)$ in $X$ is $\left(r_{1}+r_{2}\right)$-convergent to $x_{*}$ if and only if there exists a sequence $\left(y_{i}\right)$ in $X$ such that

$$
y_{i} \stackrel{r_{1}}{\rightarrow} x_{*} \quad \text { and } \quad\left\|x_{i}-y_{i}\right\| \leq r_{2}, i=1,2, \ldots
$$


Proof. Assume (3.1). $y_{i} \stackrel{r_{1}}{\rightarrow} x_{*}$ means that for all $\varepsilon>0$ there exists an $i_{\varepsilon}$ such that

$$
\left\|y_{i}-x_{*}\right\|<r_{1}+\varepsilon \text { if } i \geq i_{\varepsilon} .
$$

Since $\left\|x_{i}-y_{i}\right\| \leq r_{2}$, this yields immediately

$$
\left\|x_{i}-x_{*}\right\| \leq\left\|x_{i}-y_{i}\right\|+\left\|y_{i}-x_{*}\right\|<r_{1}+r_{2}+\varepsilon \text { if } i \geq i_{\varepsilon} .
$$

Hence, $\left(x_{i}\right)$ is $\left(r_{1}+r_{2}\right)$-convergent to $x_{*}$.

Assume now that $\left(x_{i}\right)$ is $\left(r_{1}+r_{2}\right)$-convergent to $x_{*}$. With

$$
y_{i}:= \begin{cases}x_{*} & \text { if }\left\|x_{i}-x_{*}\right\| \leq r_{2} \\ x_{i}+r_{2} \frac{x_{*}-x_{i}}{\left\|x_{*}-x_{i}\right\|} & \text { if }\left\|x_{i}-x_{*}\right\|>r_{2}\end{cases}
$$

we have

$$
\left\|y_{i}-x_{*}\right\| \leq \begin{cases}0 & \text { if }\left\|x_{i}-x_{*}\right\| \leq r_{2} \\ \left\|x_{i}-x_{*}\right\|-r_{2} & \text { if }\left\|x_{i}-x_{*}\right\|>r_{2}\end{cases}
$$

and

$$
\left\|x_{i}-y_{i}\right\| \leq r_{2} \quad \text { for } \quad i=1,2, \ldots
$$

By $(1.2), x_{*} \in \operatorname{LIM}^{r_{1}+r_{2}} x_{i}$ implies

$$
\lim \sup \left\|x_{i}-x_{*}\right\| \leq r_{1}+r_{2}
$$

and therefore

$$
\limsup \left\|y_{i}-x_{*}\right\| \leq r_{1}
$$

that means $y_{i} \stackrel{r_{1}}{\rightarrow} x_{*}$.

In particular, for $r_{1}=0$ and $r_{2}=r>0$, the latter conclusion says that a sequence $\left(x_{i}\right)$ is $r$-convergent to $x_{*}$ iff there exists a sequence $\left(y_{i}\right)$ such that

$$
y_{i} \rightarrow x_{*} \quad \text { and } \quad\left\|x_{i}-y_{i}\right\| \leq r, i=1,2, \ldots
$$

The necessity means that if $\left(x_{i}\right)$ is an approximation of a convergent sequence $y_{i} \rightarrow x_{*}$ with $r$ as maximal approximation error then it is still $r$-convergent to $x_{*}$. This is what was already said in the introduction. On the other hand, if $\left(x_{i}\right)$ is $r$-convergent to $x_{*}$ then there exists a sequence $\left(y_{i}\right)$ near $\left(x_{i}\right)$ (i.e., $\left\|x_{i}-y_{i}\right\| \leq r$ for all $i$ ) which converges (in the classical sense) to $x_{*}$.

To state an example of use of the preceding, let us consider now a special type of sequences which arise as integer part of others. It corresponds to the computational practice where a real number is approximated by the maximal machine number less or equal to the exact one.

Proposition 3.2. Assume $\left(x_{i}\right)$ is a sequence in $\left(\mathbb{R}^{n},\|\cdot\|\right)$ which converges to $x_{*}$. For $x=\left(x^{1}, x^{2}, \ldots, x^{n}\right) \in \mathbb{R}^{n}$, denote $[x]:=\left(\left[x^{1}\right],\left[x^{2}\right], \ldots,\left[x^{n}\right]\right)$, where $[\alpha]$ is the integer part of the real number $\alpha$. 
(a) If $\|\cdot\|$ is the maximum norm then

$$
x_{*} \in \operatorname{LIM}^{1}\left[x_{i}\right] \quad \text { and } \quad \operatorname{LIM}^{0.5}\left[x_{i}\right] \neq \emptyset .
$$

(b) If $\|\cdot\|$ is the Euclidean norm then

$$
x_{*} \in \operatorname{LIM}^{\sqrt{n}}\left[x_{i}\right] \quad \text { and } \quad \operatorname{LIM}^{0.5 \sqrt{n}}\left[x_{i}\right] \neq \emptyset
$$

Proof. Since $0 \leq x_{i}^{j}-\left[x_{i}^{j}\right]<1$ for all $i \in \mathbb{N}$ and $j \in\{1,2, \ldots, n\}$, we have

$$
\left\|x_{i}-\left[x_{i}\right]\right\|< \begin{cases}1 & \text { if }\|\cdot\| \text { is the maximal norm } \\ \sqrt{n} & \text { if }\|\cdot\| \text { is the Euclidean norm. }\end{cases}
$$

Therefore, Proposition 3.1 implies that

$$
x_{*} \in \operatorname{LIM}^{r} x_{i} \quad \text { for } \quad r= \begin{cases}1 & \text { if }\|\cdot\| \text { is the maximal norm } \\ \sqrt{n} & \text { if }\|\cdot\| \text { is the Euclidean norm. }\end{cases}
$$

Let $\tilde{x}_{*}:=\left[x_{*}\right]-(0.5,0.5, \ldots, 0.5)$. Since $x_{i} \rightarrow x_{*}$, there exists an $i_{*}$ such that

$$
\left[x_{*}^{j}\right]-1<x_{i}^{j}<\left[x_{*}^{j}\right]+1 \quad \text { for } j \in\{1,2, \ldots, n\}, i \geq i_{*},
$$

which yields $\left[x_{i}^{j}\right] \in\left\{\left[x_{*}^{j}\right]-1,\left[x_{*}^{j}\right]\right\}$, and therefore

$$
\left|\left[x_{i}^{j}\right]-\tilde{x}_{*}^{j}\right|=0.5 \quad \text { for } \quad j \in\{1,2, \ldots, n\}, i \geq i_{*} .
$$

Hence

$$
\left\|\left[x_{i}\right]-\tilde{x}_{*}\right\|= \begin{cases}0.5 & \text { if }\|\cdot\| \text { is the maximal norm } \\ 0.5 \sqrt{n} & \text { if }\|\cdot\| \text { is the Euclidean norm }\end{cases}
$$

for $i \geq i_{*}$. That means by definition

$$
\tilde{x}_{*} \in \operatorname{LIM}^{r}\left[x_{i}\right] \quad \text { for } \quad r= \begin{cases}0.5 & \text { if }\|\cdot\| \text { is the maximal norm } \\ 0.5 \sqrt{n} & \text { if }\|\cdot\| \text { is the Euclidean norm. }\end{cases}
$$

Note that all parameters $r$ stated in Proposition 3.2 are already optimal, i.e., one cannot give smaller parameters which are suitable for any case. To see this, just consider the following.

EXAMPLE 3.1. Let

$$
x_{i}^{1}=x_{i}^{2}=\cdots=x_{i}^{n}=(-1)^{i} / i .
$$

Then $x_{i}=\left(x_{i}^{1}, x_{i}^{2}, \ldots, x_{i}^{n}\right)$ converges to $x_{*}=(0,0, \ldots, 0)$, and

$$
\left[x_{i}\right]= \begin{cases}(0,0, \ldots, 0) & \text { if } i \text { is even } \\ -(1,1, \ldots, 1) & \text { if } i \text { is odd }\end{cases}
$$


Since

$$
\|-(1,1, \ldots, 1)-(0,0, \ldots, 0)\|= \begin{cases}1 & \text { if }\|\cdot\| \text { is the maximal norm } \\ \sqrt{n} & \text { if }\|\cdot\| \text { is the Euclidean norm }\end{cases}
$$

it is easy to see that

$$
x_{*} \notin \operatorname{LIM}^{r}\left[x_{i}\right] \quad \text { for } \quad r< \begin{cases}1 & \text { if }\|\cdot\| \text { is the maximal norm } \\ \sqrt{n} & \text { if }\|\cdot\| \text { is the Euclidean norm, }\end{cases}
$$

and

$$
\operatorname{LIM}^{r}\left[x_{i}\right]=\emptyset \quad \text { for } \quad r< \begin{cases}0.5 & \text { if }\|\cdot\| \text { is the maximal norm } \\ 0.5 \sqrt{n} & \text { if }\|\cdot\| \text { is the Euclidean norm }\end{cases}
$$

In the proof of Proposition 2.1 we have already shown that if $\left(x_{i}\right)$ converges to $x_{*}$ then $\operatorname{LIM}^{r} x_{i}=\bar{B}_{r}\left(x_{*}\right)$. This is a part of the next.

Proposition 3.3. A sequence $\left(x_{i}\right) \subset \mathbb{R}^{n}$ converges to $x_{*}$ if and only if $\operatorname{LIM}^{r} x_{i}=\bar{B}_{r}\left(x_{*}\right)$.

Proof. It remains to show that $\operatorname{LIM}^{r} x_{i}=\bar{B}_{r}\left(x_{*}\right)$ implies $x_{i} \rightarrow x_{*}$. Assume the contrary that $\left(x_{i}\right)$ has a cluster point $x_{*}^{\prime}$ different from $x_{*}$. Then the point

$$
\bar{x}_{*}:=x_{*}+\frac{r}{\left\|x_{*}-x_{*}^{\prime}\right\|}\left(x_{*}-x_{*}^{\prime}\right)
$$

satisfies

$$
\left\|\bar{x}_{*}-x_{*}^{\prime}\right\|=r+\left\|x_{*}-x_{*}^{\prime}\right\|>r .
$$

Since $x_{*}^{\prime}$ is a cluster point, this inequality implies by definition that $\bar{x}_{*} \notin \operatorname{LIM}^{r} x_{i}$, a contradiction to $\left\|\bar{x}_{*}-x_{*}\right\|=r$ and $\operatorname{LIM}^{r} x_{i}=\bar{B}_{r}\left(x_{*}\right)$. Thus $x_{*}$ is the only cluster point of $\left(x_{i}\right)$ as a bounded sequence (by Proposition 2.2) in some finite-dimensional normed space. Consequently, $\left(x_{i}\right)$ converges to $x_{*}$.

It follows directly that $x_{i} \rightarrow x_{*}$ yields the existence of two points $y_{1}, y_{2} \in$ $\operatorname{LIM}^{r} x_{i}$ satisfying $\left\|y_{1}-y_{2}\right\|=2 r$. In general, this existence does not imply the convergence of $\left(x_{i}\right)$, as the following shows.

ExAmPle 3.2. Let $\left(x_{i}\right)$ be a sequence in $\mathbb{R}^{2}$ defined by $x_{i}:=\left(\xi_{i}, 0\right), \xi_{i}=$ $(-1)^{i}$. For $\|\cdot\|$ as the maximum norm, we have

$$
\operatorname{LIM}^{1} x_{i}=\left\{(0, \eta) \in \mathbb{R}^{2}:|\eta| \leq 1\right\}
$$

Clearly, $y_{1}:=(0,1)$ and $y_{2}:=(0,-1)$ satisfy $y_{1}, y_{2} \in \operatorname{LIM}^{1} x_{i}$ and $\left\|y_{1}-y_{2}\right\|=2$, but this sequence $\left(x_{i}\right)$ does not converge at all.

But this fact changes completely in strictly convex spaces. 
Proposition 3.4. Suppose $\left(x_{i}\right)$ is a sequence in some (finite-dimensional) strictly convex space. If there are $y_{1}, y_{2} \in \operatorname{LIM}^{r} x_{i}$ satisfying $\left\|y_{1}-y_{2}\right\|=2 r$ then $\left(x_{i}\right)$ converges to $\frac{1}{2}\left(y_{1}+y_{2}\right)$.

Proof. Let $y_{3}$ be an arbitrary cluster point of $\left(x_{i}\right)$. Then $y_{1}, y_{2} \in \operatorname{LIM}^{r} x_{i}$ implies

$$
\left\|y_{1}-y_{3}\right\| \leq r \quad \text { and } \quad\left\|y_{2}-y_{3}\right\| \leq r
$$

(compare Proposition 3.5). On the other hand,

$$
2 r=\left\|y_{1}-y_{2}\right\| \leq\left\|y_{1}-y_{3}\right\|+\left\|y_{2}-y_{3}\right\| .
$$

Therefore

$$
\left\|y_{1}-y_{3}\right\|=\left\|y_{2}-y_{3}\right\|=r
$$

Since

$$
\frac{1}{2}\left(y_{2}-y_{1}\right)=\frac{1}{2}\left(\left(y_{3}-y_{1}\right)+\left(y_{2}-y_{3}\right)\right) \text { and }\left\|\frac{1}{2}\left(y_{2}-y_{1}\right)\right\|=r,
$$

the strict convexity of the normed space considered implies

$$
\frac{1}{2}\left(y_{2}-y_{1}\right)=y_{3}-y_{1}=y_{2}-y_{3} .
$$

Hence, $y_{3}=\frac{1}{2}\left(y_{1}+y_{2}\right)$. That means $\frac{1}{2}\left(y_{1}+y_{2}\right)$ is the only cluster point of $\left(x_{i}\right)$ as a bounded sequence (by Proposition 2.2) in some finite-dimensional normed space. Consequently, $\left(x_{i}\right)$ must converge to $\frac{1}{2}\left(y_{1}+y_{2}\right)$.

The previous two assertions are concerned with the relation between a convergent sequence and its $r$-limit set. In general, we do not expect sequences considered to be convergent, but to have several cluster points.

Proposition 3.5.

(a) If $c$ is a cluster point of the sequence $\left(x_{i}\right)$ then

$$
\operatorname{LIM}^{r} x_{i} \subseteq \bar{B}_{r}(c) .
$$

(b) Let $C$ be the set of cluster points of $\left(x_{i}\right) \subset \mathbb{R}^{n}$. Then

$$
\operatorname{LIM}^{r} x_{i}=\bigcap_{c \in C} \bar{B}_{r}(c)=\left\{x_{*} \in \mathbb{R}^{n}: C \subseteq \bar{B}_{r}\left(x_{*}\right)\right\} .
$$

Proof. (a) For an arbitrary cluster point $c$ of $\left(x_{i}\right)$ we have

$$
\left\|x_{*}-c\right\| \leq r \quad \text { for all } \quad x_{*} \in \operatorname{LIM}^{r} x_{i},
$$

otherwise there are infinite $x_{i}$ satisfying

$$
\left\|x_{*}-x_{i}\right\|>r+\varepsilon \quad \text { with } \quad \varepsilon:=\left(\left\|x_{*}-c\right\|-r\right) / 2>0,
$$


because $c$ is a cluster point of $\left(x_{i}\right)$, which contradicts (1.1). Hence, (3.2) must be true.

(b) The preceding yields immediately

$$
\operatorname{LIM}^{r} x_{i} \subseteq \bigcap_{c \in C} \bar{B}_{r}(c)
$$

If $y \in \cap_{c \in C} \bar{B}_{r}(c)$ then $\|y-c\| \leq r$ for all $c \in C$, which is equivalent to $C \subseteq \bar{B}_{r}(y)$, i.e.

$$
\bigcap_{c \in C} \bar{B}_{r}(c) \subseteq\left\{x_{*} \in \mathbb{R}^{n}: C \subseteq \bar{B}_{r}\left(x_{*}\right)\right\}
$$

If $y \notin \operatorname{LIM}^{r} x_{i}$ then, by definition, there is an $\varepsilon>0$ such that there exist infinite $x_{i}$ satisfying $\left\|x_{i}-y\right\| \geq r+\varepsilon$, which implies the existence of a cluster point $c$ of $\left(x_{i}\right)$ with $\|y-c\| \geq r+\varepsilon$, i.e., $C \nsubseteq \bar{B}_{r}(y)$ and $y \notin\left\{x_{*} \in \mathbb{R}^{n}: C \subseteq \bar{B}_{r}\left(x_{*}\right)\right\}$. Hence, $y \in \operatorname{LIM}^{r} x_{i}$ follows from $y \in\left\{x_{*} \in \mathbb{R}^{n}: C \subseteq \bar{B}_{r}\left(x_{*}\right)\right\}$, i.e.

$$
\left\{x_{*} \in \mathbb{R}^{n}: C \subseteq \bar{B}_{r}\left(x_{*}\right)\right\} \subseteq \operatorname{LIM}^{r} x_{i} .
$$

The three inclusions proved above ensure that (3.3) holds true.

As an example, consider again the sequence $\left(x_{i}\right)$ with $x_{i}=\left((-1)^{i}, 0\right) \in \mathbb{R}^{2}$. Since it only has two cluster points $(-1,0)$ and $(1,0)$, it follows from (3.3) that $\operatorname{LIM}^{r} x_{i}=\bar{B}_{r}((-1,0)) \cap \bar{B}_{r}((1,0))$.

(3.3) shows that an $r$-limit set of $\left(x_{i}\right)$ is "almost round" in such a sense that its boundary consists of $|C|$ boundary parts of balls with radius $r$, where $|C|$ denotes the cardinality of the cluster point set $C$ of $\left(x_{i}\right)$.

Let us consider the relation of rough convergence to set convergence in SetValued Analysis. Recall if $\left(K_{i}\right)_{i \in \mathbb{N}}$ is a sequence of subsets of a metric space $X$ then the subsets

$$
\begin{aligned}
& \operatorname{Limsup}_{i \rightarrow \infty} K_{i}:=\left\{x \in X \mid \liminf _{i \rightarrow \infty} d\left(x, K_{i}\right)=0\right\}, \\
& \operatorname{Liminf}_{i \rightarrow \infty} K_{i}:=\left\{x \in X \mid \lim _{i \rightarrow \infty} d\left(x, K_{i}\right)=0\right\}
\end{aligned}
$$

are called upper or lower limit of the sequence $\left(K_{i}\right)$ (see [1, p.17]).

By definition, Limsup $\left\{x_{i}\right\}$ is the set of cluster points of $\left(x_{i}\right)$. Therefore, it follows from (3.4)

$$
\text { Limsup }\left\{x_{i}\right\} \subset \bar{B}_{r}\left(x_{*}\right) \text { for all } x_{*} \in \operatorname{LIM}^{r} x_{i},
$$

and from $(3.3)$

$$
\operatorname{LIM}^{r} x_{i}=\bigcap_{c \in \operatorname{Limsup}\left\{x_{i}\right\}} \bar{B}_{r}(c)
$$


if the normed space considered is finite-dimensional.

Proposition 3.6.

$$
\operatorname{LIM}^{r} x_{i}=\operatorname{Liminf} \bar{B}_{r}\left(x_{i}\right) .
$$

Proof. a) Assume $y \in \operatorname{LIM}^{r} x_{i}$. Define then

$$
y_{i}:= \begin{cases}x_{i}+\frac{r}{\left\|y-x_{i}\right\|}\left(y-x_{i}\right) & \text { if }\left\|y-x_{i}\right\|>r \\ y & \text { otherwise. }\end{cases}
$$

Since

$$
\left\|x_{i}+\frac{r}{\left\|y-x_{i}\right\|}\left(y-x_{i}\right)-y\right\|=\left|\frac{r}{\left\|y-x_{i}\right\|}-1\right| \cdot\left\|y-x_{i}\right\|=\left|\left\|y-x_{i}\right\|-r\right|
$$

we have

$$
\left\|y_{i}-y\right\|= \begin{cases}\left\|y-x_{i}\right\|-r & \text { if }\left\|y-x_{i}\right\|>r \\ 0 & \text { otherwise. }\end{cases}
$$

Therefore, $y \in \operatorname{LIM}^{r} x_{i}$ yields that $y_{i}$ tends to $y$ as $i \rightarrow \infty$. But $\left\|x_{i}-y_{i}\right\| \leq r$, i.e., $y_{i} \in \bar{B}_{r}\left(x_{i}\right)$. Consequently, $\lim _{i \rightarrow \infty} d\left(y, \bar{B}_{r}\left(x_{i}\right)\right)=0$, which means by definition that $y \in \operatorname{Liminf} \bar{B}_{r}\left(x_{i}\right)$.

b) If $y \in \operatorname{Liminf} \bar{B}\left(x_{i}, r\right)$ then, by definition, there exists a sequence $\left(y_{i}\right)$ satisfying $y_{i} \rightarrow y$ and $y_{i} \in B_{r}\left(x_{i}\right)$, i.e., $\left\|x_{i}-y_{i}\right\| \leq r$. Therefore, Proposition 3.1 implies $y \in \operatorname{LIM}^{r} x_{i}$.

\section{Dependence on Roughness Degree}

The previous section is concerned with properties of $r$-limit set for a fixed

roughness degree $r$. Let us now investigate the dependence of $r$-limit $\operatorname{LIM}^{r} x_{i}$ of a fixed sequence $\left(x_{i}\right)$ on varying parameter $r$.

It follows from definition

$$
\operatorname{LIM}^{r_{1}} x_{i} \subseteq \operatorname{LIM}^{r_{2}} x_{i} \text { if } r_{1}<r_{2}
$$

This monotonicity is included in the following.

Proposition 4.1. Suppose $r \geq 0$ and $\sigma>0$. Then

(a) $\operatorname{LIM}^{r} x_{i}+\bar{B}_{\sigma}(0) \subseteq \operatorname{LIM}^{r+\sigma} x_{i}$.

(b) $\bar{B}_{\sigma}(y) \subseteq \operatorname{LIM}^{r} x_{i}$ implies $y \in \operatorname{LIM}^{r-\sigma} x_{i}$.

Proof. (a) Let $y \in \operatorname{LIM}^{r} x_{i}$ and $z \in \bar{B}_{\sigma}(0)$. By definition, for all $\varepsilon>0$ there exists an $i_{\varepsilon}$ such that $\left\|x_{i}-y\right\|<r+\varepsilon$ if $i \geq i_{\varepsilon}$, which implies by $\|z\| \leq \sigma$ that

$$
\left\|x_{i}-y-z\right\|<r+\sigma+\varepsilon \quad \text { if } \quad i \geq i_{\varepsilon} .
$$


Hence, $y+z \in \operatorname{LIM}^{r+\sigma} x_{i}$. point

(b) Let $c$ be an arbitrary cluster point of $\left(x_{i}\right)$. If $\|y-c\|>r-\sigma$ then the

$$
x_{*}:=y+\frac{\sigma}{\|y-c\|}(y-c)
$$

satisfies

$$
\left\|x_{*}-c\right\|=\sigma+\|y-c\|>\sigma+(r-\sigma)=r .
$$

By (3.2), this yields $x_{*} \notin \operatorname{LIM}^{r} x_{i}$, a contradiction to $\left\|x_{*}-y\right\|=\sigma$ and $\bar{B}_{\sigma}(y) \subseteq$ $\operatorname{LIM}^{r} x_{i}$. Hence, $\|y-c\| \leq r-\sigma$ for all cluster points $c \in C$. Consequently, it follows from (3.3)

$$
y \in \bigcap_{c \in C} \bar{B}_{r-\sigma}(c)=\operatorname{LIM}^{r-\sigma} x_{i} .
$$

In general, $\operatorname{LIM}^{r} x_{i}+\bar{B}_{\sigma}(0) \neq \operatorname{LIM}^{r+\sigma} x_{i}$ is possible. For instance, the sequence $\left(x_{i}\right)$ in the two-dimensional Euclidean space with

$$
x_{i}=\left(0, \xi_{i}\right) \in \mathbb{R}^{2}, \xi_{i}=(-1)^{i}, i=1,2, \ldots
$$

satisfies

$$
\operatorname{LIM}^{0.5} x_{i}+\bar{B}_{0.5}(0)=\emptyset+\bar{B}_{0.5}(0)=\emptyset \neq\{(0,0)\}=\operatorname{LIM}^{1} x_{i} .
$$

The reason does not consist in the voidness of $\operatorname{LIM}^{0.5} x_{i}$, because

$$
\operatorname{LIM}^{1} x_{i}+\bar{B}_{1}(0)=\{(0,0)\}+\bar{B}_{1}(0)=\bar{B}_{1}(0)
$$

while $\left\|(\sqrt{3}, 0)-x_{i}\right\|=\|(\sqrt{3}, \pm 1)\|=2$ for all $i$ implies

$$
(\sqrt{3}, 0) \in \operatorname{LIM}^{2} x_{i} \backslash \bar{B}_{1}(0),
$$

i.e., $\operatorname{LIM}^{1} x_{i}+\bar{B}_{1}(0) \neq \operatorname{LIM}^{2} x_{i}$.

Define

$$
\bar{r}:=\inf \left\{r \in \mathbb{R}_{+}: \operatorname{LIM}^{r} x_{i} \neq \emptyset\right\} .
$$

By the monotonicity given in (4.1), we have

$$
\operatorname{LIM}^{r} x_{i} \begin{cases}=\emptyset & \text { for } r<\bar{r} \\ \neq \emptyset & \text { for } r>\bar{r} .\end{cases}
$$

Moreover, by Proposition 4.1, for all $r>\bar{r}$ and $\sigma \in(0, r-\bar{r}), \operatorname{LIM}^{r} x_{i}$ always contains some ball with radius $\sigma$, that means at least

$$
\operatorname{int}\left(\operatorname{LIM}^{r} x_{i}\right) \neq \emptyset \text { for } r>\bar{r} .
$$


Therefore,

$$
\operatorname{int}\left(\operatorname{LIM}^{r} x_{i}\right)=\emptyset \text { implies } r \leq \bar{r} \text { and } \operatorname{LIM}^{r^{\prime}} x_{i}=\emptyset \text { for } r^{\prime} \in[0, r)
$$

It remains to ask what properties the $\bar{r}$-limit has.

Proposition 4.2.

(a) $r=\bar{r}$ if and only if

$$
\operatorname{LIM}^{r} x_{i} \neq \emptyset \text { and } \operatorname{int}\left(\operatorname{LIM}^{r} x_{i}\right)=\emptyset
$$

(b) If $(X,\|\cdot\|)$ is a finite-dimensional strictly convex space then $r=\bar{r}$ if and only if $\mathrm{LIM}^{r} x_{i}$ is a singleton.

Proof. (a) Let $r=\bar{r}$. We have to show (4.6). It follows from Proposition 4.3 proved later that $\operatorname{LIM}^{\bar{r}} x_{i}=\bigcap_{r^{\prime}>\bar{r}} \operatorname{LIM}^{r^{\prime}} x_{i}$. For $r^{\prime}>\bar{r}, \operatorname{LIM}^{r^{\prime}} x_{i}$ is nonempty (by (4.3)) and closed (Proposition 2.4). By (4.1), we have

$$
\bigcap_{r^{\prime}>\bar{r}} \operatorname{LIM}^{r^{\prime}} x_{i}=\bigcap_{\bar{r}<r^{\prime} \leq \bar{r}+1} \operatorname{LIM}^{r^{\prime}} x_{i}
$$

and $\operatorname{LIM}^{r^{\prime}} x_{i}, r^{\prime} \in(\bar{r}, \bar{r}+1]$ is a family of nonempty closed subsets in the compact set $\mathrm{LIM}^{\bar{r}+1} x_{i}$ having the finite intersection property. Therefore, their intersection is nonempty (see [2, p. 69]). Hence $\operatorname{LIM}^{\bar{r}} x_{i} \neq \emptyset$.

If $\operatorname{int}\left(\operatorname{LIM}^{r} x_{i}\right) \neq \emptyset$ then it contains some ball $\bar{B}_{\sigma}(y)$ with $\sigma>0$, and by Proposition 4.1 we have $\operatorname{LIM}^{r-\sigma} x_{i} \neq \emptyset$, i.e., $r>\bar{r}$. Thus $r=\bar{r}$ yields $\operatorname{int}\left(\operatorname{LIM}^{r} x_{i}\right)=\emptyset$.

Assume (4.6). Since $\operatorname{LIM}^{r} x_{i} \neq \emptyset$, we have $r \geq \bar{r}$. On the other hand, by (4.5), $r \leq \bar{r}$ follows from int $\left(\operatorname{LIM}^{r} x_{i}\right)=\emptyset$. Consequently, $r=\bar{r}$.

(b) If $\operatorname{LIM}^{r} x_{i}$ is a singleton then (4.6) is fulfilled. Therefore, by (a), $r=\bar{r}$. It remains to show that $\operatorname{LIM}^{\bar{r}} x_{i}$ is a singleton. This follows directly from its strict convexity (by Proposition 2.5), $\operatorname{LIM}^{\bar{r}} x_{i} \neq \emptyset$, and $\operatorname{int}\left(\operatorname{LIM}^{\bar{r}} x_{i}\right)=\emptyset$.

Consider again the sequence $\left(x_{i}\right)$ with $x_{i}=\left((-1)^{i}, 0\right) \in \mathbb{R}^{2}$. (3.3) yields $\operatorname{LIM}^{1} x_{i}=\bar{B}_{1}((-1,0)) \cap \bar{B}_{1}((1,0))$. For both maximal and Euclidean norm, we have

$$
(0,0) \in \operatorname{LIM}^{1} x_{i} \neq \emptyset \text { and } \operatorname{int}\left(\operatorname{LIM}^{1} x_{i}\right)=\emptyset
$$

which implies by Proposition 4.2 that the minimal convergence degree of this sequence is $\bar{r}=1$. In particular, if $\|\cdot\|$ is the maximal norm then

$$
\operatorname{LIM}^{1} x_{i}=\left\{(0, \eta) \in \mathbb{R}^{2}:|\eta| \leq 1\right\}
$$

This shows that the strict convexity of the space considered is really necessary for $\operatorname{LIM}^{\bar{r}} x_{i}$ to be a singleton. 
Conversely, the uniqueness of $\bar{r}$-limit point is no signal for the strict convexity of the space. For instance, consider $\left(x_{i}\right) \subset \mathbb{R}^{2}$ with

$$
x_{i}= \begin{cases}(0,1) & \text { if } i=4 j+1 \\ (1,0) & \text { if } i=4 j+2 \\ (0,1) & \text { if } i=4 j+3 \\ (0,1) & \text { if } i=4(j+1)\end{cases}
$$

$(j=0,1, \ldots)$. Then for maximal norm, we also have $\operatorname{LIM}^{1} x_{i}=\{(0,0)\}$, although with this norm the space is not strictly convex.

Proposition 4.3.

$$
\operatorname{cl}\left(\bigcup_{0 \leq r^{\prime}<r} \operatorname{LIM}^{r^{\prime}} x_{i}\right) \subseteq \operatorname{LIM}^{r} x_{i}=\bigcap_{r^{\prime}>r} \operatorname{LIM}^{r^{\prime}} x_{i} .
$$

If $r \neq \bar{r}$ then $\bigcup_{0 \leq r^{\prime}<r} \operatorname{LIM}^{r^{\prime}} x_{i}=\operatorname{LIM}^{r} x_{i}$.

Proof. By the monotonicity given in (4.1) and the closedness of $r$-limit (Proposition 2.4) we have

$$
\operatorname{cl}\left(\bigcup_{0 \leq r^{\prime}<r} \operatorname{LIM}^{r^{\prime}} x_{i}\right) \subseteq \operatorname{LIM}^{r} x_{i} \subseteq \bigcap_{r^{\prime}>r} \operatorname{LIM}^{r^{\prime}} x_{i} .
$$

Consider now an arbitrary $y \in X \backslash \operatorname{LIM}^{r} x_{i}$. By definition, there is an $\varepsilon>0$ such that

$$
\forall k \in \mathbb{N} \quad \exists i \geq k:\left\|x_{i}-y\right\| \geq r+\varepsilon .
$$

This implies for $r^{\prime}<r+\varepsilon$ that $\varepsilon^{\prime}:=r+\varepsilon-r^{\prime}>0$ and

$$
\forall k \in \mathbb{N} \quad \exists i \geq k:\left\|x_{i}-y\right\| \geq r^{\prime}+\varepsilon^{\prime}
$$

Thus $y \notin \operatorname{LIM}^{r^{\prime}} x_{i}$ for $r^{\prime}<r+\varepsilon$, which implies $y \notin \bigcap_{r^{\prime}>r} \operatorname{LIM}^{r^{\prime}} x_{i}$. Hence $\operatorname{LIM}^{r} x_{i}=\bigcap_{r^{\prime}>r} \operatorname{LIM}^{r^{\prime}} x_{i}$.

For $r<\bar{r}$, it is clear that

$$
\operatorname{cl}\left(\bigcup_{0 \leq r^{\prime}<r} \operatorname{LIM}^{r^{\prime}} x_{i}\right)=\operatorname{LIM}^{r} x_{i}=\emptyset .
$$

Let $r=r_{1}>\bar{r}$ and $r_{0}=\left(\bar{r}+r_{1}\right) / 2$. Since $r_{0}>\bar{r}$ we can choose a $y_{0} \in \operatorname{LIM}^{r_{0}} x_{i} \neq \emptyset$. Consider an arbitrary $y_{1} \in \operatorname{LIM}^{r_{1}} x_{i}$. Proposition 2.5 yields

$$
y_{\lambda}=(1-\lambda) y_{0}+\lambda y_{1} \in \operatorname{LIM}^{(1-\lambda) r_{0}+\lambda r_{1}} x_{i}, \text { for } \lambda \in[0,1] \text {. }
$$

Consequently,

$$
y_{\lambda} \in \bigcup_{0 \leq r^{\prime}<r} \operatorname{LIM}^{r^{\prime}} x_{i} \text { for } \lambda \in[0,1)
$$


Since

$$
\left\|y_{\lambda}-y_{1}\right\|=(1-\lambda)\left\|y_{0}-y_{1}\right\| \rightarrow 0 \text { as } \lambda \rightarrow 1
$$

it follows

$$
y_{1} \in \operatorname{cl}\left(\bigcup_{0 \leq r^{\prime}<r} \operatorname{LIM}^{r^{\prime}} x_{i}\right) .
$$

Hence

$$
\operatorname{cl}\left(\bigcup_{0 \leq r^{\prime}<r} \operatorname{LIM}^{r^{\prime}} x_{i}\right)=\operatorname{LIM}^{r} x_{i}
$$

holds true for $r>\bar{r}$, too.

Let us investigate the semi-continuity of the set-valued mapping

$$
F: \mathbb{R}_{+} \rightarrow 2^{X} \quad \text { with } \quad F(r):=\operatorname{LIM}^{r} x_{i}
$$

Due to Bouligand [4] and Kuratowski [6] (compare also [1,2]), $F$ is said to be lower semi-continuous (l.s.c.) at $r$, if for each open set $U$ satisfying $F(r) \cap U \neq \emptyset$ there exists a neighborhood $V(r)$ such that

$$
t \in V(r) \text { implies } F(t) \cap U \neq \emptyset \text {. }
$$

It is called upper semi-continuous (u.s.c.) at $r$ if for each open set $U$ containing $F(r)$ there is a neighborhood $V(r)$ such that

$$
t \in V(r) \text { implies } F(t) \subset U \text {. }
$$

We say $F$ is l.s.c. or u.s.c. on $I$ if it has the corresponding property at every $r \in I$.

Proposition 4.4. F is l.s.c. on $(\bar{r},+\infty)$ and u.s.c. on $[\bar{r},+\infty)$.

Proof. Let $r>\bar{r}$ and $U$ be an open subset satisfying $F(r) \cap U \neq \emptyset$. Since $F(r)=\operatorname{LIM}^{r} x_{i}$ is convex (by Proposition 2.5) and $\operatorname{int}\left(\operatorname{LIM}^{r} x_{i}\right) \neq \emptyset$ (by (4.4)), $\operatorname{int}\left(\operatorname{LIM}^{r} x_{i}\right) \cap U$ is nonempty and open. Therefore, there exists a ball $\bar{B}_{\sigma}(y)$ contained in $\operatorname{int}\left(\operatorname{LIM}^{r} x_{i}\right) \cap U \subset \operatorname{LIM}^{r} x_{i}$. By Proposition 4.1, it follows $y \in$ $\operatorname{LIM}^{r-\sigma} x_{i} \cap U$. Thus (4.1) implies

$$
y \in \operatorname{LIM}^{r-\sigma} x_{i} \cap U \subseteq \operatorname{LIM}^{t} x_{i} \cap U=F(t) \cap U \quad \text { for } \quad t \in(r-\sigma,+\infty) .
$$

Hence, $F$ is l.s.c. at each $r \in(\bar{r},+\infty)$.

Assume now $r \geq \bar{r}$ and $U$ is an open subset satisfying $F(r) \subset U$. By (4.1),

$$
F(t)=\operatorname{LIM}^{t} x_{i} \subseteq \operatorname{LIM}^{r} x_{i}=F(r) \subset U \text { for } t \in[0, r] .
$$

Consequently, we only have to prove that there is a $\delta>0$ such that

$$
F(t)=\operatorname{LIM}^{t} x_{i} \subset U \quad \text { for } \quad t \in(r, r+\delta) .
$$


If this does not hold true, then there exists a decreasing sequence $\left(t_{j}\right)$ such that

$$
\lim t_{j}=r \quad \text { and } \quad \operatorname{LIM}^{t_{j}} x_{i} \backslash U \neq \emptyset, j=1,2, \ldots
$$

Clearly,

$$
\bigcap_{t>r} \operatorname{LIM}^{t} x_{i} \subseteq \bigcap_{j \in \mathbb{N}} \operatorname{LIM}^{t_{j}} x_{i}
$$

If $y \notin \bigcap_{t>r} \operatorname{LIM}^{t} x_{i}$ then there exists $\bar{t}>r$ such that $y \notin \operatorname{LIM}^{\bar{t}} x_{i}$. By $\lim t_{j}=r$ and (4.1), there is a $t_{\bar{j}}<\bar{t}$ such that $y \notin \operatorname{LIM}^{t_{\bar{j}}} x_{i}$, which yields $y \notin \bigcap_{j \in \mathbb{N}} \operatorname{LIM}^{t_{j}} x_{i}$. Hence

$$
\bigcap_{t>r} \operatorname{LIM}^{t} x_{i}=\bigcap_{j \in \mathbb{N}} \operatorname{LIM}^{t_{j}} x_{i}
$$

Therefore, Proposition 4.3 and $\operatorname{LIM}^{r} x_{i} \subset U$ imply

$$
\bigcap_{j \in \mathbb{N}}\left(\operatorname{LIM}^{t_{j}} x_{i} \backslash U\right)=\left(\bigcap_{j \in \mathbb{N}} \operatorname{LIM}^{t_{j}} x_{i}\right) \backslash U=\operatorname{LIM}^{r} x_{i} \backslash U=\emptyset .
$$

But $\operatorname{LIM}^{t_{j}} x_{i} \backslash U, j=1,2, \ldots$, form a decreasing sequence of nonempty closed subsets contained in the compact set $\operatorname{LIM}^{t_{1}} x_{i}$, therefore their intersection is not empty (compare [2, p. 69]). This contradiction completes our proof.

\section{Rough Cauchy Sequences}

It is well know that each convergent sequence in a normed space satisfies Cauchy condition, and conversely, each Cauchy sequence in a Banach space converges. The relation between roughly convergent sequences and rough Cauchy sequences cannot be described so shortly.

Let $\left(x_{i}\right)$ be $r$-convergent, i.e., $\operatorname{LIM}^{r} x_{i} \neq \emptyset$. Take an arbitrary $x_{*} \in \operatorname{LIM}^{r} x_{i}$. By definition, for all $\varepsilon>0$ there exists an $i_{\varepsilon} \in \mathbb{N}$ such that $i, j \geq i_{\varepsilon}$ implies $\left\|x_{i}-x_{*}\right\| \leq r+\varepsilon / 2$ and $\left\|x_{j}-x_{*}\right\| \leq r+\varepsilon / 2$, which yields immediately

$$
\left\|x_{i}-x_{j}\right\| \leq\left\|x_{i}-x_{*}\right\|+\left\|x_{j}-x_{*}\right\| \leq 2 r+\varepsilon
$$

Hence $\left(x_{i}\right)$ is a $\rho$-Cauchy sequence with $\rho=2 r$. This Cauchy degree cannot be generally decreased. Indeed, let $z \in \mathbb{R}^{n}$ with $\|z\|=r$ and $x_{i}=(-1)^{i} z$, then the sequence $\left(x_{i}\right)$ is $r$-convergent with $0 \in \operatorname{LIM}^{r} x_{i}$, and $\rho=2 r$ is its minimal Cauchy degree.

Conversely, let $\rho \geq 0$ be a Cauchy degree of some given sequence $\left(x_{i}\right)$. Can we expect its convergence degree to equal $\rho / 2$, i.e., $\operatorname{LIM}^{\rho / 2} x_{i} \neq \emptyset$ ? Not always. The main purpose of this section is to find a possibly small convergence degree of given $\rho$-Cauchy sequences. 
First, let us formulate some properties of Cauchy sequences. Similarly to the boundedness of roughly convergent sequences (Proposition 2.2), the following is rather obvious and its proof is therefore omitted.

Proposition 5.1. A sequence $\left(x_{i}\right)$ is bounded if and only if there exists an $\rho \geq 0$ such that it is a $\rho$-Cauchy sequence.

As $\left(x_{i}\right)$ is a bounded sequence in a finite-dimensional normed space, the cluster point set $C$ is nonempty and bounded. Thus its diameter $D(C)$ and the radius $R(C)$ of its circumscribed ball are finite.

Recall that the diameter $D(S)$ and the radius $R(S)$ of the circumscribed ball of a bounded subset $S$ in some normed space $\left(\mathbb{R}^{n},\|\cdot\|\right)$ is defined as follows:

$$
D(S):=\sup _{x, y \in S}\|x-y\|, \quad R(S):=\inf _{x \in \mathbb{R}^{n}} \sup _{y \in S}\|x-y\| .
$$

Proposition 5.2. Let $C$ be the cluster point set of the sequence $\left(x_{i}\right)$. Then $D(C)$ is the minimal Cauchy degree and $R(C)$ is the minimal convergence degree $\bar{r}$ of $\left(x_{i}\right)$. That means

$$
D(C)=\min \left\{\rho \in \mathbb{R}_{+}:\left(x_{i}\right) \text { is a } \rho \text {-Cauchy sequence }\right\},
$$

and

$$
\operatorname{LIM}^{r} x_{i} \begin{cases}=\emptyset & \text { for } r<R(C) \\ \neq \emptyset & \text { for } r \geq R(C)\end{cases}
$$

Proof. (a) If $0 \leq \rho<D(C)$ then for $\varepsilon=(D(C)-\rho) / 3$ there exist two cluster points $c_{1}$ and $c_{2}$ such that $\left\|c_{1}-c_{2}\right\|>\rho+2 \varepsilon$. For all $k \in \mathbb{N}$ there are $i_{1}, i_{2} \geq k$ such that

$$
\left\|x_{i_{1}}-c_{1}\right\|<\varepsilon / 2 \quad \text { and } \quad\left\|x_{i_{2}}-c_{2}\right\|<\varepsilon / 2
$$

which imply

$$
\begin{aligned}
\left\|x_{i_{1}}-x_{i_{2}}\right\| & \geq\left\|c_{1}-c_{2}\right\|-\left\|\left(x_{i_{1}}-c_{1}\right)-\left(x_{i_{2}}-c_{2}\right)\right\| \\
& \geq\left\|c_{1}-c_{2}\right\|-\left(\left\|x_{i_{1}}-c_{1}\right\|+\left\|x_{i_{2}}-c_{2}\right\|\right) \\
& >\rho+2 \varepsilon-(\varepsilon / 2+\varepsilon / 2) \\
& =\rho+\varepsilon .
\end{aligned}
$$

That means $\left(x_{i}\right)$ is not a $\rho$-Cauchy sequence if $0 \leq \rho<D(C)$.

Assume now $\rho \geq D(C)$ and $\varepsilon>0$ is arbitrary. Clearly, there are only finite $x_{i}$ outside $C+B_{\varepsilon / 2}(0)$, otherwise there exists a cluster point outside $C+B_{\varepsilon / 2}(0)$, because the space considered is finite-dimensional and $C+B_{\varepsilon / 2}(0)$ is open. Therefore, there is an $i_{\varepsilon}$ such that $x_{i} \in C+B_{\varepsilon / 2}(0)$ for $i \geq i_{\varepsilon}$. That means if $i_{1} \geq i_{\varepsilon}$ and $i_{2} \geq i_{\varepsilon}$ then $\left\|x_{i_{1}}-c_{1}\right\|<\varepsilon / 2$ and $\left\|x_{i_{2}}-c_{2}\right\|<\varepsilon / 2$ for some $c_{1}, c_{2} \in C$, which yields by $\left\|c_{1}-c_{2}\right\| \leq D(C) \leq \rho$

$$
\begin{aligned}
\left\|x_{i_{1}}-x_{i_{2}}\right\| & \leq\left\|c_{1}-c_{2}\right\|+\left\|x_{i_{1}}-c_{1}\right\|+\left\|x_{i_{2}}-c_{2}\right\| \\
& <\rho+\varepsilon / 2+\varepsilon / 2 \\
& =\rho+\varepsilon .
\end{aligned}
$$


Hence, $\left(x_{i}\right)$ is a $\rho$-Cauchy sequence if $\rho \geq D(C)$. Thus (5.2) is proved.

(b) If $r<R(C)$, by (5.1), for all $z \in \mathbb{R}^{n}$ there exists $y \in C$ such that $\|z-y\|>r$, which implies by Proposition 3.5 that $z \notin \operatorname{LIM}^{r} x_{i}$, i.e., $\operatorname{LIM}^{r} x_{i}=\emptyset$.

If $r>R(C)$, by (5.1), there is an $x_{*} \in \mathbb{R}^{n}$ such that $\left\|x_{*}-y\right\| \leq r$ for all $y \in C$, which yields $x_{*} \in \bigcap_{y \in C} \bar{B}_{r}(y)$. Hence, by Proposition 3.5, $\operatorname{LIM}^{r} x_{i} \neq \emptyset$.

It follows now from (4.2) that $R(C)=\bar{r}$. Therefore, by Proposition 4.2, $\operatorname{LIM}^{r} x_{i} \neq \emptyset$ for $r=\bar{r}=R(C)$. Thus (5.3) is completely proved.

The latter proposition shows that $R(C)$ is the minimal convergence degree and $D(C)$ is the minimal Cauchy degree of $\left(x_{i}\right)$. To find the relation between convergence degree and Cauchy degree of a sequence, this result gives us a hint to use the relation between the radius of the circumscribed ball of a bounded set and its diameter, which is given in the following.

Proposition 5.3. (H. E. W. Jung [5], see also [8, p. 78]) For any bounded closed subset $S$ of the Euclidean space $\left(\mathbb{R}^{n},\|\cdot\|\right)$, the following inequality holds between the diameter $D(S)$ of $S$ and the radius $R(S)$ of the circumscribed ball:

$$
R(S) \leq \sqrt{\frac{n}{2(n+1)}} D(S)
$$

The inequality (5.4) becomes an equality if and only if conv $S$ contains a regular simplex with side length $D(S)$.

Proposition 5.4. (Bohnenblust [3]) Let $S$ be a bounded closed subset of some normed space $\left(\mathbb{R}^{n},\|\|.\right)$. Then the following inequality holds between the diameter $D(S)$ of $S$ and the radius $R(S)$ of the circumscribed ball:

$$
R(S) \leq \frac{n}{(n+1)} D(S)
$$

Note that Bohnenblust formulated this result in Minkowski geometry. Leichtweiss [7] showed later that $n /(n+1)$ is the best factor by showing cases where the equality holds true.

Let us state a relation between Cauchy degree and convergence degree.

Proposition 5.5. Let $\left(x_{i}\right)$ be a $\rho$-Cauchy sequence in some normed space $\left(\mathbb{R}^{n},\|\cdot\|\right)$ for some $\rho \geq 0$. Then it is $r$-convergent for $r \geq \frac{n}{n+1} \rho$. In particular, if $\|\cdot\|$ is the Euclidean norm, then $\left(x_{i}\right)$ is $r$-convergent for $r \geq \sqrt{\frac{n}{2(n+1)}} \rho$.

Proof. Since $\left(x_{i}\right)$ is a $\rho$-Cauchy sequence, by Proposition 5.2 , we have $\rho \geq$ $D(C)$, where $C$ is the cluster point set of $\left(x_{i}\right)$. Therefore, by Proposition 5.4

$$
r \geq \frac{n}{n+1} \rho \text { implies } \quad r \geq \frac{n}{n+1} D(C) \geq R(C) .
$$

Consequently, by Proposition 5.2 again, $\operatorname{LIM}^{r} x_{i} \neq \emptyset$ if $r \geq \frac{n}{n+1} \rho$. 
In particular, if $\|\cdot\|$ is the Euclidean norm, then we obtain the remaining assertion by using Proposition 5.3 instead of Proposition 5.4.

Remark. The parameter $n$ given in Proposition 5.5 is the dimension of the normed space considered. We can obtain a better result by replacing $n$ through the dimension $m$ of the cluster point set $C$ of $\left(x_{i}\right)$ (i.e., of the affine hull of $C$ ), because $m$ is often smaller than $n$, and in this case we have

$$
\frac{n}{n+1}>\frac{m}{m+1} \quad \text { and } \quad \sqrt{\frac{n}{2(n+1)}}>\sqrt{\frac{m}{2(m+1)}}
$$

To conclude this section, we consider the rough limit in some proper subsets of $\mathbb{R}^{n}$ of $\rho$-Cauchy sequences.

If $\left(x_{i}\right)$ is a $\rho$-Cauchy sequence, then for all $r>\rho$ there exists a $k \in \mathbb{N}$ such that

$$
\left\{x_{i}: i \geq k\right\} \subset \operatorname{LIM}^{\left(x_{i}\right), r} x_{i} .
$$

In particular, for $\left(x_{i}\right)$ as a Cauchy sequence in the classical sense (i.e., $\rho=0$ ) and for all $r>0$, there exists a $k \in \mathbb{N}$ such that (5.5) holds. Of course, if $\left(x_{i}\right)$ is a sequence in some subset $M$, then $\operatorname{LIM}^{\left(x_{i}\right), r} x_{i} \subseteq \operatorname{LIM}^{M, r} x_{i}$ implies that

$$
\operatorname{LIM}^{M, r} x_{i} \neq \emptyset \quad \text { for } \quad r>\rho
$$

although $M$ is possibly not closed. This property is also available for smaller $r$. The following gives an example in Euclidean spaces.

Proposition 5.6. Let $M$ be a convex (and not necessarily closed) subset of the Euclidean space $\left(\mathbb{R}^{n},\|\cdot\|\right)$, and $\left(x_{i}\right)$ be a $\rho$-Cauchy sequence in $M$ (for instance $\left.M=\operatorname{conv}\left\{x_{i}: i \in \mathbb{N}\right\}\right)$. Then

$$
\operatorname{LIM}^{M, r} x_{i} \neq \emptyset \quad \text { for } \quad r \geq \sqrt{\frac{n}{2(n+1)}} \rho
$$

Proof. Consider the cluster point set $C$ of $\left(x_{i}\right)$. Clearly, both $C$ and its convex hull conv $C$ are closed and bounded. It is well known that $C$ has a center $z \in \mathbb{R}^{n}$ of its circumscribed ball, i.e.,

$$
\sup _{y \in C}\|z-y\|=R(C)=\inf _{x \in \mathbb{R}^{n}} \sup _{y \in C}\|x-y\|,
$$

which is unique and belongs to $\operatorname{conv} C$ (compare [8, p. 78]). It follows $C \subseteq$ $B_{R(C)}(z)$, which yields by (3.3) that $z \in \operatorname{LIM}^{R(C)} x_{i}$. Moreover, since $C$ is the cluster point set of $\left(x_{i}\right) \subset M$, we have $C \subset \operatorname{cl} M$. Therefore, $z \in \operatorname{cl} M$ follows from the convexity of $M$ and $z \in \operatorname{conv} C$. 
If $r>R(C)$ then, for $\sigma=r-R(C)>0$,

$$
\bar{B}_{\sigma}(z) \subseteq \operatorname{LIM}^{R(C)+\sigma} x_{i}=\operatorname{LIM}^{r} x_{i}
$$

Thus $z \in \operatorname{cl} M$ yields

$$
\emptyset \neq M \cap \bar{B}_{\sigma}(z) \subseteq M \cap \operatorname{LIM}^{r} x_{i}=\operatorname{LIM}^{M, r} x_{i}
$$

If $R(C) \geq r \geq \sqrt{\frac{n}{2(n+1)}} \rho$ then Proposition 5.2 and Proposition 5.3 imply

$$
R(C) \geq r \geq \sqrt{\frac{n}{2(n+1)}} \rho \geq \sqrt{\frac{n}{2(n+1)}} D(C) \geq R(C) .
$$

Consequently,

$$
r=R(C)=\sqrt{\frac{n}{2(n+1)}} \rho=\sqrt{\frac{n}{2(n+1)}} D(C) .
$$

By Proposition 5.3, this equality is only fulfilled if $C$ contains $\left\{c_{1}, c_{2}, \ldots, c_{n+1}\right\}$ with $\left\|c_{j}-c_{k}\right\|=D(C)$ for $j \neq k$. Since $z$ is the center of the circumscribed ball of the regular simplex $\operatorname{conv}\left\{c_{1}, c_{2}, \ldots, c_{n+1}\right\}$, we have

$$
z=\frac{1}{n+1} \sum_{j=1}^{n+1} c_{j} \quad \text { and } \quad z \in \operatorname{int}\left(\operatorname{conv}\left\{c_{1}, c_{2}, \ldots, c_{n+1}\right\}\right)
$$

Consequently, since $\left\{c_{1}, c_{2}, \ldots, c_{n+1}\right\} \subseteq C \subseteq \operatorname{cl} M$ and $M$ is convex, $z$ must belong to $M$. Therefore, we finally have

$$
z \in M \cap \operatorname{LIM}^{R(C)} x_{i}=M \cap \operatorname{LIM}^{r} x_{i}=\operatorname{LIM}^{M, r} x_{i}
$$

\section{Concluding Remarks}

Although it is assumed that the normed space considered is finite-dimensional, some of the results presented in this paper remains true in infinite-dimensional spaces, for instance Propositions 2.1-2.4. Some other conclusions are also valid in infinite-dimensional spaces under certain compactness assumption. 
This paper was written while the author was visiting the Abdus Salam International Centre for Theoretical Physics, Trieste, Italy, as an Associate. A generous grant from the Swedish International Development Cooperation Agency facilitated the visit. The author would like to express his sincere gratitude to both bodies.

\section{REFERENCES}

1. J.-P. Aubin and H. Frankowska, "Set-Valued Analysis," Birkhäuser, Boston Basel Berlin, 1990.

2. C. Berge, "Topological Spaces," Oliver \& Boyd, Edinburgh and London, 1963.

3. F. Bohnenblust, Convex regions and projections in Minkowski spaces, Ann. of Math. 39 (1938), 301-308.

4. G. Bouligand, Sur la Semi-Continuité d'Inclusions et Quelques Sujets Connexes, Ens. Math. 31 (1932), 14-22.

5. H. E. W. Jung, Über die kleinste Kugel, die eine räumliche Figur einschließt, J. Reine Angew. Math. 123 (1901), 241-257.

6. K. Kuratowski, Les Fonctions Semi-Continues dans l'Espace des Ensembles Fermés, Fund. Math. 18 (1932), 148-159.

7. K. Leichtweiss, Zwei Extremalprobleme der Minkowski-Geometrie, Math. Z. 62 (1955) 37-49.

8. K. Leichtweiss, "Konvexe Mengen", Springer-Verlag, Berlin Heidelberg New York, 1980. 